\section{Thermal Decomposition along Dislocations}

$\mathrm{J}_{\mathrm{ACH}}{ }^{1}$ reports observations that lead him to suppose that the thermal decomposition solid $\rightarrow$ solid plus gas occurs preferentially along dislocation lines. Perhaps the following observations lend support to this view.

Investigating the behaviour of thin plate-like crystals of cadmium iodids in the beam of an electron-microseope, in the same manner as did Forty ${ }^{2}$ with lead iodide, we observe phenomena generally similar to those reported by him. Fig. 1 shows a partly decomposed crystal (ef. Forty's Fig. 12). Such a picture is obtained by having the electron beam slightly off centre and sufficiently intense for 'white-patches' to develop and grow, the crystal thon being slowly consumed, and the 'decomposition edge' movo slowly across the field of view (Fig. 1 from bottom left to top right). The white-patches are presumably similar to those reported by Forty in the case of lead iodide. We would regard these white-patches (whether they grow by a defeet mechanism within the crystal (as suggested by Forty), or are increasingly deep etch-pits, or whatever) as "centres of decomposition" in so far as it is their growth and coaleseing which result in the crystal being consumed and hence the decomposition-edge moving forward. These white-patches differ from those reportod by Forty in lead iodide in that none has ever been seen to move through the crystal, but generally only to grow. Exceptionally a patch will contract and vanish although its neighbours continue to grow.

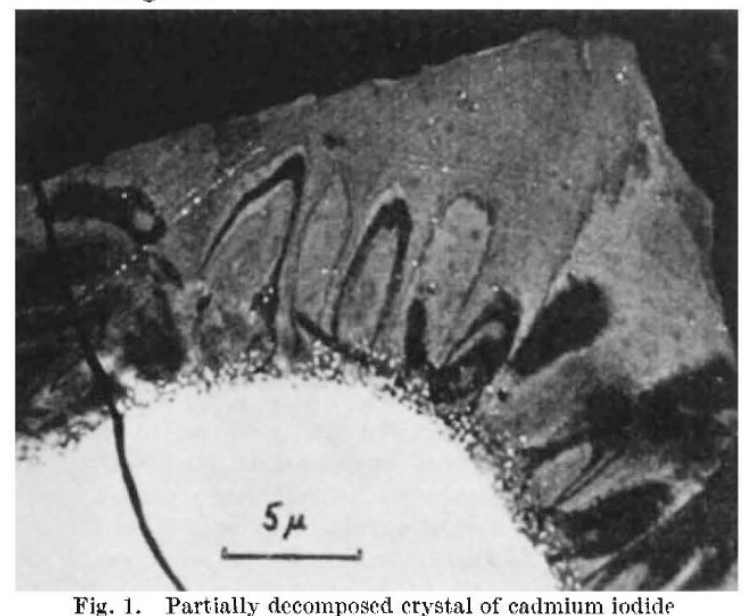

Fig. 1. Partially decomposed crystal of cadmium iodide

Generally the patches appear at random but occasionally in lines as shown in Fig. 1. Since there is nothing to indicate a change in specimen thickness (such as at a growth step) or a crystal boundary we take it that the patches mark dislocation lines or planes along which families of dislocations, extending between the crystal faces, lie. We also notice that after such a crystal is completely destroyed only a very thin structureless film, which we assume to be a carbon film deposited on the specimen in the microscope, is left on which there are very faint dark lines (rather like faint pencil lines) where the lines of white-patches occurred. We take these faint lines to be due to heavy metal impurities occurring preferentially at dislocation lines.

We suggest that these observations indicate preferential decomposition at dislocations, and probably along disloeation lines, the white-patches being etch-pits deepening rapidly to give holes right through the erystals ${ }^{3}$; the activation onergy for decomposition being lowered at and near a dislocation line.

J. R. Bristow

Department of Applied Physics,

B. L. REES

Welsh College of Advanced Technology, Cardiff.

${ }^{1}$ Jach, J., Nature, 196, 827 (1962).

${ }^{2}$ Forty, A. J., Phil. Mag., 5, 787 (1960).

${ }^{3}$ Horn, F. H., Phil. Maty., 43, 1210 (1952).

\section{CHEMISTRY}

\section{Reflectance Spectra of Some Mercury (II) Compounds on Active Adsorbents}

THE well-documented hypsochromic and bathochromic shifts observed in the wave-length maxima of the transmittance spectra of many solutes can be explained in many cases on the basis of solute-solvent interactions in which the polarity of the solvent and/or solute is an important factor in determining the type and magnitude of the shift $^{1,2}$.

An investigation utilizing the technique of spectral reflectance was undertaken to ascertain whether shifts observed in the wave-length maxima of the diffuse reflectance spectra of solid-solid systems are subject to the same general principles which govern solute-solvent systems and thus can be interproted according to accepted polarization theory.

The model systems examined consisted of an optically absorbing solid species and an adsorbent. Two series of mercury (II) compounds containing anions of known relative polarizability were selected: (1) the iodide, bromide, and chloride; (2) the sulphide and oxide. The adsorbents used were neutral Woelm alumina, silica gel, and sodium fluoride.

The two powdered components were mixed under con. trolled and anhydrous conditions and permitted to interact at room temperature for varying lengths of time. The diffuse ultra-violet and visible reflectance spectra were measured with a recording $D K-2$ spectrophotometer equipped with an integrating sphere reflectance attachment. The absorbance was plotted as a function of wavelength. Spectral data in the form of the wave-length maxima $\left(\lambda_{\max }\right)$ of the principal optical absorption peaks are given in Table 1 .

The $\lambda_{\max }$ (initial) were obtained immediately after mixing with the adsorbent whilo the $\lambda_{\max }$ (final) were measured 60-90 days later when interaction between the two components had apparently ceased. The $\lambda_{\max }$ (initial) are considered to bo thoso of tho absorbing species unmodified by the adsorbent whereas displacements shown by a comparison of the $\lambda_{\max }$ (final) and $\lambda_{\max }$ (initial) are believed to arise as a result of polarization and adsorption of the absorbing species by the adsorbent.

In the case of the halides in the unadsorbed state, in accordance with prediction, the iodide absorbed farthest in the red followed in turn by the bromide and chloride. The order $\left(\lambda_{\max }\right)$ for the two chaleogenides was sulphide $(570 \mathrm{~m} \mu)$ and oxide $(501 \mathrm{~m} \mu)$.

All displacements in $\lambda_{\max }$ observed to occur with time were bathochromic. The relative magnitudes of the shifts arc believed to be dependent primarily on tho polariza. bility of the adsorbed species and the polarizing power of the adsorbent. In all cases the halides were adsorbed to a greater extent and exhibited sharper $\lambda_{\max }$ peaks than the chalcogenides.

For a series of anions with the same charge, it should be expected that the polarizability would increase with the size of the ion. As applied to the solid-solid systems the increasing order of polarizability should be chloride. bromide, iodide for the first series and oxide, sulphide for the second. Spectrally, this should be indicated by an increase in the bathochromic shift as polarization and adsorption occur. The experimental data in Table 1 support this prediction. However, the mercuric iodidealumina system is considered anomalous ${ }^{3,4}$.

As would be expocted, mercuric iodido was most affected and was rapidly adsorbed on silica gel and alumina. On silica gel the red iodide was converted to a very pale opalescent yellow. Spectrally, the $568 \mathrm{~m} \mu$ peak disappeared completely while the band in the ultra-violet underwent a displacement of approximately $50 \mathrm{~m} \mu$ during adsorption. In the mercuric iodide-alumina system the peak in the visible was lost during adsorption while no 